Editorial

\title{
Primary frozen shoulder can it be treated with hydrodilatation
}

\author{
Volume 9 Issue 3 - 2017

\section{Mohammed Waseem} \\ Consultant upperlimb and Trauma Surgeon, Honorary Senior \\ Lecturer, Edge Hill University Ormskirk, UK
}

\author{
Correspondence: Mohammed Waseem, Macclesfield \\ General Hospital,Victoria Road, Macclesfield SK 10 3BL, UK, Tel \\ 1625661315, Email m.waseem@nhs.net
}

Received: October 23, 2017 | Published: November 16, 2017

There were 7 patients from the local anaesthetic group who required sedation and a second procedure. In the sedation group, one patient had a 2 nd procedure.

Overall, patients who underwent hydrodilatation under sedation have a better satisfaction and improved faster post-procedure. In our study, hydrodilatation under sedation is a better option; however, both choices should be discussed formally with patients during consultations prior to hydrodilatation.

We are currently undetaking a study between ultrasound guided hydro dilation and image intensifier guided technique. We have also added an additional hyloraunic acid injection group which might reduce recurrence. The recurrence remains higher in patients with type I diabetes and with poor compliance.

\section{Acknowledgments}

None.

\section{Conflicts of interest}

None. of motion (ROM), visual analogue scale (VAS) for pain and Oxford Shoulder Score (OSS).

Range of motion improved in $80 \%$ of patients who underwent hydrodilatation with a mean VAS score of 3 and OSS of 19 at 36 weeks. This was achieved in the sedation group at 6 weeks. 